\title{
Human papillomavirus, cervical cancer and preventive vaccines
}

\section{Editorial}

There are more than 100 human papillomavirus (HPV) subtypes, of which at least 13 subtypes are cancer-causing or high-risk subtypes. HPV is mainly transmitted through sexual contact the majority of the persons are infected with HPV shortly after the onset of sexual activity. HPV subtypes 16 and 18 cause around $70 \%$ of cervical cancers and precancerous cervical lesions. Cancers of the vulva, vagina, penis, and anus are also linked with HPV. In 2012, about 84 $\%$ of the globally new cases with cervical cancer that is the second most common cancer in women lived in less developed areas. HPV infection is most common in sexually active young women, 18 to 30 years of age with a sharp decrease in prevalence after 30 years of age. Nevertheless, cervical cancer is more common in women older than 35 years. Around 270,000 women died from cervical cancer and more than $85 \%$ of these deaths occur in low- and middle-income countries.

HPV have been also detected in a wide variety of animals. Eightyfive HPV genotypes of more than 200 subtypes are well characterized. Additional 120 isolates are partially characterized potentially new genotypes. HPVs can infect both basal epithelial cells of the skin and inner lining of tissues, thus, HPVs can be categorized as cutaneous types and mucosal types. The mucosal types infect the tissue lining of the mouth, throat, respiratory tract, or anogenital epithelium. HPVs can be classified into high-risk and low-risk HPV subtypes, based on their association with cervical cancer and precancerous lesions. The high-risk HPV subtypes include subtypes 16, 18, 31, 33, 34, 35, 39, $45,51,52,56,58,59,66,68$, and 70 , whereas the low-risk subtypes are subtypes $6,11,42,43$, and 44 . HPV is associated with a variety of clinical conditions that range from innocuous lesions to cancer. Most HPV infections are benign conditions. HPV was first recognized as the cause of cutaneous wart (flat warts, common warts, plantar warts) with spontaneous resolution within 1 to 5 years. High-risk cervical intraepithelial neoplasia is commonly associated with HPV subtypes $6,11,16,18,31,33,34,35,39,42,44,45,51,52,56,58$, and 66 , while subtypes 16 and 18 are the most common. HPV subtypes 16, 18, $31,33,35,39,45,51,52,56,58,66,68$, and 70 , while subtypes 16 and 18 are the most common. PV is a relatively small, nonenveloped virus, and $55 \mathrm{~nm}$ in diameter. Its icosahedral capsid composes of 72 capsomers, which contain at least two capsid proteins, L1 and L2. Each capsomer is a pentamer of the major capsid protein, L1, while each viron capsid contains about 12 copies. The HPV genome consists of a single molecule of double-stranded, circular DNA containing around 7,900 base pairs that are associated with histones. All open reading frame $(\mathrm{ORF})$ protein-coding sequences are restricted to one strand.

Clinical manifestations of sexually transmitted infections can lead to three possible results. The first is anogenital warts (condyloma accuminatum) that are generally associated with HPV subtypes 6 and 11 and do not lead to cancer. The second is latent or inactive infection. HPV DNA is detected in around $10 \%$ of women with
Volume 5 Issue 7 - 2017

\author{
Attapon Cheepsattayakorn, ${ }^{1,2,3}$ Ruangrong \\ Cheepsattayakorn ${ }^{4}$ \\ 'Editor-in-Chief, Journal of Human Virology and Retrovirology, \\ USA \\ ${ }^{2}$ IOth Zonal Tuberculosis and Chest Disease Center,Thailand \\ ${ }^{3} 5$ th Office of Disease Prevention and Control, Ratchaburi, \\ Department of Disease Control, Ministry of Public Health, \\ Thailand \\ ${ }^{4}$ Department of Pathology, Faculty of Medicine, Chiang Mai \\ University, Thailand
}

Correspondence: Attapon Cheepsattayakorn, I0th Zonal Tuberculosis and Chest Disease Center, 143 Sridornchai Road Changklan Muang Chiang Mai 50I00 Thailand,Tel 6653 140767, 6653 276364, Fax 6653 140773, 6653 273590,

Email attapon1958@gmail.com, attaponche@yahoo.com

Received: November 24, 2017 | Published: November 24, 2017

cytologically normal cervical epithelium. The third is active infection, which is associated with high-risk HPV subtypes in which the virus causes changes in infected cells which may result in vaginal, vulvar, cervical, penile, urethral, or urinary bladder intraepithelial neoplasia. Some prospective studies demonstrated that around $28 \%$ of women in whom HPV DNA was detected developed squamous intraepithelial lesions within 2years., compared to 1 to $3 \%$ of women in whom HPV DNA was not detected. The risk for progression for HPV subtypes 16 and 18 was greater (around $40 \%$ ) than for other HPV subtypes. Diagnosis of HPV cervical infection can be made by the conventional cytology, the Papanicolaou-stained (Pap) smear that was introduced in 1949. The Pap smear procedure has false-negative rates as high as 20 to $30 \%$. The other molecularly diagnostic tests are HPV DNA detection by type-specific polymerase chain reaction (PCR), general primer PCR, and liquid hybridization and HPV mRNA detection.

Use of latex condoms and spermicide may decrease the risk of contracting HPV. Nevertheless, condom are not totally reliable, because HPV may be contracted by contact with other parts of the body. Three HPV vaccines are currently approved by the United States Food and Drug Administration (US FDA) that can prevent infections with HPV subtypes 16 and 18 . Two vaccines are approved for use in females ages 9 to 26 years including males in the same age range, while another vaccine is approved for use in males ages 9 to 15 years. In October 2016, the US FDA approved a 2-dose schedule for boys and girls initiating vaccination at ages 9 to 14 years and the second dose are to be administered 6 to 12 months after the first. 
Those initiating the HPV vaccination series at older ages, including teens who begin getting vaccinated after they turn 15 years old or who are immunocompromized should be vaccinated according to the 3-dose schedule. These three vaccines provide nearly $100 \%$ anogenital cancer protection. In conclusion, the optimal vaccines should contain a cocktail of virus-liked particles (VLPs), which are empty virus capsid containing the major HPV capsid antigen and possibly the minor capsid antigen but lacking viral DNA.

\section{Acknowledgments}

None.

\section{Conflicts of interest}

None. 